\title{
STUDY OF A LEAST-SQUARES-BASED ALGORITHM FOR AUTOREGRESSIVE SIGNALS SUBJECT TO WHITE NOISE
}

\author{
WEI XING ZHENG
}

Received 16 October 2002

A simple algorithm is developed for unbiased parameter identification of autoregressive (AR) signals subject to white measurement noise. It is shown that the corrupting noise variance, which determines the bias in the standard least-squares (LS) parameter estimator, can be estimated by simply using the expected LS errors when the ratio between the driving noise variance and the corrupting noise variance is known or obtainable in some way. Then an LS-based algorithm is established via the principle of bias compensation. Compared with the other LS-based algorithms recently developed, the introduced algorithm requires fewer computations and has a simpler algorithmic structure. Moreover, it can produce better AR parameter estimates whenever a reasonable guess of the noise variance ratio is available.

\section{Introduction}

Estimation of the parameters of autoregressive (AR) signals from noisy measurements has been an important topic of research in the field of signal processing $[2,4,6]$. Since the standard least-squares (LS) method is unable to produce unbiased estimates of the AR parameters in the presence of noise, many identification algorithms have been developed with a view to achieving unbiasedness in AR signal estimation; for instance, the modified Yule-Walker (MYW) equations method [1], the maximum likelihood (ML) method [7], the recursive prediction error (RPE) method [3], the modified least-squares (MLS) method [5], and the improved least-squares (ILS) methods [8, 9]. It is of interest to note that the ILS-type algorithms are built on the simple idea of estimating the variance of the corrupting noise in an efficient way and then removing the noise-induced bias from the standard LS estimator in a straightforward way so as to attain unbiased AR parameter estimates. The good performances of the ILS-type algorithms are as follows. Firstly, as a linear regression-based method, the ILS-types methods require much less numerical efforts than the ML method, the RPE method, and the MLS method. Secondly, the ILS-type algorithms not only are well suited for online estimation, but also have much better numerical robustness than the MYW method. Thirdly, unlike the ML method and 
the MYW method, the ILS-type algorithms can simultaneously estimate the corrupting noise variance and the signal power that may be required in certain signal processing applications.

The objective of the present paper is to develop a simple algorithm for unbiased parameter identification of AR signals subject to white measurement noise. Note that the assumption on the measurement noise is a restriction, but it is not unrealistic. Like the other ILS-type algorithms, central to this new algorithm is the estimation of the corrupting noise variance, which determines the bias in the LS parameter estimator. However, it is observed that the other ILS algorithms need to compute some extra autocovariance estimates for the purpose of getting an estimate of the corrupting noise variance. This apparently requires added computations. In this paper, it is assumed that the ratio between the AR driving noise variance and the corrupting noise variance is given or obtainable in some way. Note that, on the one hand, this assumption may be considered as restrictive in some practical situations since it may be difficult to have information on both the driving noise and the corrupting one simultaneously. On the other hand, however, it may still conform to a number of signal processing application cases. For example, in speech processing, the level of background noise relative to a speech signal is sometimes predictable beforehand according to the experience so that a reasonable description of the noisy scenario (or the noise variance ratio) is admissible [4]. Under the imposed assumption, the corrupting noise variance can be estimated by simply using the expected LS errors. Then a new LS-based algorithm is established via the principle of bias compensation. Compared with the other ILS-type algorithms, the developed algorithm requires fewer computations and has a simpler algorithmic structure. Moreover, it can produce better AR parameter estimates once a sensible conjecture of the noise variance ratio is given. The sensitivity of the developed algorithm with respect to the noise variance ratio is also studied via computer simulations.

\section{Signal model}

Assume that the AR signal $x(t)$ is generated by a model of the form

$$
x(t)=\sum_{i=1}^{p} a_{i} x(t-i)+v(t),
$$

where $p$ is the order of the model, $v(t)$ is the driving (white) noise with zero mean and finite variance $\sigma_{v}^{2}$, and $\left\{a_{i}, i=1, \ldots, p\right\}$ are the AR parameters.

Let

$$
y(t)=x(t)+w(t)
$$

be a noisy measurement of the AR signal, where $w(t)$ is the corrupting (white) noise with zero mean and finite variance $\sigma_{w}^{2}$.

The noisy AR model, which consists of (2.1) and (2.2), can be expressed in a vector form as

$$
y(t)=\mathbf{y}_{t}^{\top} \mathbf{a}+\epsilon(t)
$$


where

$$
\mathbf{a}^{\top}=\left[a_{1} \cdots a_{p}\right]
$$

is the parameter vector which contains the $p$ parameters of the AR signal, and

$$
\mathbf{y}_{t}^{\top}=[y(t-1) \cdots y(t-p)]
$$

is the regression vector which contains the $p$ delayed noisy measurements of the AR signal. Moreover, in (2.3), $\epsilon(t)$ is the equation error which is defined by

$$
\epsilon(t)=v(t)+w(t)-\mathbf{w}_{t}^{\top} \mathbf{a},
$$

where

$$
\mathbf{w}_{t}^{\top}=[w(t-1) \cdots w(t-p)]
$$

\section{LS estimation and analysis}

The objective of noisy AR signal identification is to estimate the AR parameters $\left\{a_{i}\right.$, $i=1, \ldots, p\}$, including the driving noise variance $\sigma_{v}^{2}$ and the corrupting noise variance $\sigma_{w}^{2}$, from a sample of $N$ noisy measurements $\{y(t), t=1, \ldots, N\}$.

To solve this parameter estimation problem, several assumptions are needed. First, the signal order $p$ is assumed to be known. Second, the driving noise $v(t)$ and the corrupting noise $w(t)$ are statistically uncorrelated. Note that the first assumption may be relaxed so that only an upper bound of $p$ is given, whereas the second assumption can easily be satisfied in practical circumstances.

The standard LS parameter estimation is based on minimizing the mean squared error criterion

$$
J(\mathbf{a})=E\left[\epsilon(t)^{2}\right]
$$

which gives rise to the LS estimate of $\mathbf{a}$ (see [1]):

$$
\mathbf{a}_{\mathrm{LS}}=\mathbf{R}^{-1} \mathbf{r},
$$

where

$$
\mathbf{R}=E\left[\mathbf{y}_{t} \mathbf{y}_{t}^{\top}\right], \quad \mathbf{r}=E\left[\mathbf{y}_{t} y(t)\right]
$$

To analyze the asymptotic property of $\mathbf{a}_{\mathrm{LS}}$, a regression vector of the (noise-free) AR signal $x(t)$ is introduced:

$$
\mathbf{x}_{t}^{\top}=[x(t-1) \cdots x(t-p)] .
$$

With (2.5), (2.7), and (3.4), the noisy measurement equation (2.2) may be rewritten in a vector form as

$$
\mathbf{y}_{t}=\mathbf{x}_{t}+\mathbf{w}_{t}
$$


96 Algorithm for autoregressive signals subject to noise

Following the assumptions that $v(t)$ and $w(t)$ are white noises and are mutually uncorrelated, it is straightforward to derive

$$
\begin{aligned}
E\left[\mathbf{x}_{t} \epsilon(t)\right] & =E\left[\mathbf{x}_{t} v(t)\right]+E\left[\mathbf{x}_{t} w(t)\right]-E\left[\mathbf{x}_{t} \mathbf{w}_{t}\right] \mathbf{a} \\
& =\mathbf{0}+\mathbf{0}-\mathbf{0} \mathbf{a} \\
& =\mathbf{0} \\
E\left[\mathbf{w}_{t} \epsilon(t)\right] & =E\left[\mathbf{w}_{t} v(t)\right]+E\left[\mathbf{w}_{t} w(t)\right]-E\left[\mathbf{w}_{t} \mathbf{w}_{t}\right] \mathbf{a} \\
& =\mathbf{0}+\mathbf{0}-\sigma_{w}^{2} \mathbf{I}_{p} \mathbf{a} \\
& =-\sigma_{w}^{2} \mathbf{a},
\end{aligned}
$$

where $\mathbf{I}_{p}$ is an identity matrix of order $p$. By means of (3.5) and (3.6), it is easy to get

$$
\begin{aligned}
E\left[\mathbf{y}_{t} \epsilon(t)\right] & =E\left[\mathbf{x}_{t} \epsilon(t)\right]+E\left[\mathbf{w}_{t} \epsilon(t)\right] \\
& =\mathbf{0}-\sigma_{w}^{2} \mathbf{a} \\
& =-\sigma_{w}^{2} \mathbf{a} .
\end{aligned}
$$

Equation (3.7) shows that $E\left[\mathbf{y}_{t} \epsilon(t)\right]$ is not a zero vector, that is, $\epsilon(t)$ is no longer orthogonal to the projection space spanned by $\mathbf{y}_{t}$ due to the presence of the corrupting noise $w(t)$. In fact, substituting (2.2) and (3.7) into (3.2) immediately yields

$$
\mathbf{a}_{\mathrm{LS}}=\mathbf{a}+\Delta \mathbf{a}, \quad \Delta \mathbf{a}=-\sigma_{w}^{2} \mathbf{R}^{-1} \mathbf{a} .
$$

The above asymptotic expression for $\mathbf{a}_{\mathrm{LS}}$ clearly shows that $\mathbf{a}_{\mathrm{LS}}$ is biased, and the bias $\Delta \mathbf{a}$ is determined by the corrupting noise variance $\sigma_{w}^{2}$.

\section{Unbiased parameter estimation}

By using the principle of bias compensation, an unbiased estimate of the AR parameter vector a can be obtained as follows:

$$
\mathbf{a}=\mathbf{a}_{L S}-\Delta \mathbf{a}
$$

However, the bias $\Delta \mathbf{a}$ still remains unknown unless the corrupting noise variance $\sigma_{w}^{2}$ is given or may be estimated in some way.

To this end, it is necessary to take a close look at the expected LS errors

$$
J\left(\mathbf{a}_{\mathrm{LS}}\right)=E\left[\xi^{2}\left(t, \mathbf{a}_{\mathrm{LS}}\right)\right],
$$

where the LS error $\xi\left(t, \mathbf{a}_{\mathrm{LS}}\right)$ is defined by

$$
\xi\left(t, \mathbf{a}_{\mathrm{LS}}\right)=y(t)-\mathbf{y}_{t}^{\top} \mathbf{a}_{\mathrm{LS}}
$$

As shown in [8], $J\left(\mathbf{a}_{\mathrm{LS}}\right)$ is expressible as

$$
J\left(\mathbf{a}_{\mathrm{LS}}\right)=\sigma_{v}^{2}+\sigma_{w}^{2}\left(1+\mathbf{a}_{\mathrm{LS}}^{\top} \mathbf{a}\right) .
$$


The above asymptotic expression shows that the driving noise variance $\sigma_{v}^{2}$ and the corrupting noise variance $\sigma_{w}^{2}$ are closely related to each other. If one of them is known, the other is immediately obtainable. In $[8,9]$, it is shown that the corrupting noise variance $\sigma_{w}^{2}$ may be first estimated by using some extra autocovariances of $y(t)$.

In this paper, in order to implement the bias compensation procedure (4.1), it is proposed to assume that the ratio between the driving noise variance $\sigma_{v}^{2}$ and the corrupting noise variance $\sigma_{w}^{2}$, namely,

$$
\kappa^{2}=\frac{\sigma_{v}^{2}}{\sigma_{w}^{2}}
$$

is given or a proper estimate of it is available. As explained in Section 1, although this assumption may be considered as a restrictive condition in some practical situations, it may still conform to a number of signal processing application cases. For example, in quite a number of practical situations, it is possible to know that the corrupting noise just accounts for a fraction of the signal power, so that a priori information of the ratio $\kappa^{2}$ may be readily available. Further, this assumption greatly simplifies the estimation problem.

Given this assumption, substitution of (4.5) into (4.4) gives rise to

$$
J\left(\mathbf{a}_{\mathrm{LS}}\right)=\sigma_{w}^{2}\left(\kappa^{2}+1+\mathbf{a}_{\mathrm{LS}}^{\top} \mathbf{a}\right) .
$$

This immediately reveals that the corrupting noise variance $\sigma_{w}^{2}$ can be estimated by using the following equation:

$$
\sigma_{w}^{2}=\frac{J\left(\mathbf{a}_{\mathrm{LS}}\right)}{\kappa^{2}+1+\mathbf{a}_{\mathrm{LS}}^{\top} \mathbf{a}} .
$$

By means of (4.1), (4.2), and (4.7), a new ILS algorithm may be proposed for unbiased parameter identification of AR signals subject to white measurement noise. This is called the ILSR algorithm as it assumes the known ratio $\kappa^{2}$.

\section{The ILSR Algorithm}

Step 0. Initialization.

(1) Make the standard LS estimation of the AR parameter vector a:

$$
\hat{\mathbf{a}}_{\mathrm{LS}}=\hat{\mathbf{R}}_{N}^{-1} \hat{\mathbf{r}}_{N},
$$

where the autocovariance estimates $\hat{\mathbf{R}}_{N}$ and $\hat{\mathbf{r}}_{N}$ are calculated from the noisy observations $\{y(1), \ldots, y(N)\}$ as

$$
\hat{\mathbf{R}}_{N}=\frac{1}{N} \sum_{t=1}^{N} \mathbf{y}_{t} \mathbf{y}_{t}^{\top}, \quad \hat{\mathbf{r}}_{N}=\frac{1}{N} \sum_{t=1}^{N} \mathbf{y}_{t} y(t) .
$$

(2) Make estimation of the expected LS errors $J\left(\mathbf{a}_{\mathrm{LS}}\right)$ :

$$
\hat{J}_{N}\left(\hat{\mathbf{a}}_{\mathrm{LS}}\right)=\frac{1}{N} \sum_{t=1}^{N}\left(y(t)-\mathbf{y}_{t}^{\top} \hat{\mathbf{a}}_{\mathrm{LS}}\right)^{2} .
$$

(3) Set $k=0$ and $\hat{\mathbf{a}}_{\mathrm{ILS}}(0)=\hat{\mathbf{a}}_{\mathrm{LS}}$. 
Step 1. Make estimation of the corrupting noise variance $\sigma_{w}^{2}$ :

$$
\hat{\sigma}_{w}^{2}(k)=\frac{\hat{J}_{N}\left(\hat{\mathbf{a}}_{\mathrm{LS}}\right)}{\kappa^{2}+1+\hat{\mathbf{a}}_{\mathrm{LS}}^{\top} \hat{\mathbf{a}}_{\mathrm{ILS}}(k-1)} .
$$

Step 2. Make the ILS estimation of the AR parameter vector a:

$$
\hat{\mathbf{a}}_{\mathrm{ILS}}(k)=\hat{\mathbf{a}}_{\mathrm{LS}}+\hat{\sigma}_{w}^{2}(k) \mathbf{R}_{N}^{-1} \hat{\mathbf{a}}_{\mathrm{ILS}}(k-1) .
$$

Step 3. Make estimation of the driving noise variance $\sigma_{v}^{2}$ :

$$
\hat{\sigma}_{v}^{2}(k)=\kappa^{2} \hat{\sigma}_{w}^{2}(k) .
$$

Step 4. If the stop criterion

$$
\frac{\left\|\hat{\mathbf{a}}_{\mathrm{ILS}}(k)-\hat{\mathbf{a}}_{\mathrm{ILS}}(k-1)\right\|}{\left\|\hat{\mathbf{a}}_{\mathrm{ILS}}(k)\right\|}<\delta,
$$

where $\delta$ is a small positive number, is satisfied, output $\hat{\mathbf{a}}_{\mathrm{ILS}}(k), \hat{\sigma}_{v}^{2}(k)$, and $\hat{\sigma}_{w}^{2}(k)$ and stop; otherwise, set $k=k+1$ and go to Step 1 .

The consistent convergence of the proposed algorithm can be established in a similar way to that for the other ILS-type algorithms (see $[8,9])$. Moreover, it is easy to see that the ILSR algorithm can retain the advantages of the ILS-type algorithms over the MYW method, the ML method, the RPE method, and the MLS method as stated before.

A comparison is now made between the developed ILSR algorithm and the other ILStype algorithms. First, the ILSR algorithm has a better estimation accuracy than the other ILS-type algorithms. Second, since the developed algorithm does not need to compute any extra autocovariance estimates (except $\mathbf{R}, \mathbf{r}$, and $J\left(\mathbf{a}_{\mathrm{LS}}\right)$ ), it is more computationally attractive than the other ILS-type algorithms. Third, the ILSR algorithm has a simpler and more compact algorithmic structure than the other ILS-type algorithms, which enables easier implementation. Fourth, however, the other ILS-type algorithms are workable without the assumption of the known ratio $\kappa^{2}$ of the noise variances, thus having a wider domain of application than the ILSR algorithm.

\section{Numerical illustrations}

Computer simulations have been conducted for empirical assessment of the performance of the ILSR algorithm, in comparison with the standard LS method, the MYW method, the ML method, the ILSNP algorithm [8], and the ILSD algorithm [9] in terms of accuracy and computational complexity. The accuracy is described by bias and variance, while the computational complexity is measured approximately by the Matlab code flops. For an overall description of the performance, the relative error (RE) and the normalized root mean squared error (RMSE) are introduced, respectively, as follows:

$$
\mathrm{RE}=\frac{\|\mathbf{m}(\hat{\mathbf{a}})-\mathbf{a}\|}{\|\mathbf{a}\|}, \quad \operatorname{RMSE}=\sqrt{\frac{1}{M} \sum_{m=1}^{M} \frac{\left\|\hat{\mathbf{a}}_{m}-\mathbf{a}\right\|^{2}}{\|\mathbf{a}\|^{2}}},
$$


where $\mathbf{m}(\hat{\mathbf{a}})$ represents the sample mean of an estimator $\hat{\mathbf{a}}$, and $\hat{\mathbf{a}}_{m}$ stands for an estimator of $\mathbf{a}$ in the $m$ th test over a total of $M$ Monte Carlo tests.

The example used for illustration is a fourth-order AR signal as in (2.1) and (2.2). The AR parameters were selected as

$$
a_{1}=1.352, \quad a_{2}=-1.338, \quad a_{3}=0.662, \quad a_{4}=-0.24,
$$

while the noise variances were chosen as

$$
\sigma_{v}^{2}=1.0, \quad \sigma_{w}^{2}=0.38
$$

So the signal-to-noise ratio (SNR) is set approximately at $10 \mathrm{~dB}$. To examine how a priori information on the noise variance ratio $\kappa^{2}$ will affect the behavior of the ILSR algorithm, the following eleven guessed values of $\kappa^{2}$ were used:

$$
\begin{array}{ll}
\hat{\kappa}_{0}^{2}=2.6316, & \\
\hat{\kappa}_{a}^{2}=2.2, & \hat{\kappa}_{f}^{2}=2.7, \\
\hat{\kappa}_{b}^{2}=2.3, & \hat{\kappa}_{g}^{2}=2.8, \\
\hat{\kappa}_{c}^{2}=2.4, & \hat{\kappa}_{h}^{2}=2.9, \\
\hat{\kappa}_{d}^{2}=2.5, & \hat{\kappa}_{i}^{2}=3.0, \\
\hat{\kappa}_{e}^{2}=2.6, & \hat{\kappa}_{j}^{2}=3.1 .
\end{array}
$$

Note that $\hat{\kappa}_{0}^{2}$ corresponds to the case when the noise variance ratio $\kappa^{2}$ is exactly known, while $\hat{\kappa}_{a}^{2}, \ldots, \hat{\kappa}_{j}^{2}$ describe the cases when an exact knowledge of $\kappa^{2}$ is not available. In particular, $\hat{\kappa}_{a}^{2}, \ldots, \hat{\kappa}_{e}^{2}$ show that $\kappa^{2}$ is underestimated, with an estimation error ranging from more serious $16.4 \%$ in $\hat{\kappa}_{a}^{2}$ to smaller $1.2 \%$ in $\hat{\kappa}_{e}^{2}$. Similarly, $\hat{\kappa}_{f}^{2}, \ldots, \hat{\kappa}_{j}^{2}$ shows that $\kappa^{2}$ is overestimated, with an estimation error ranging from smaller $2.6 \%$ in $\hat{\kappa}_{f}^{2}$ to more serious $17.8 \%$ in $\hat{\kappa}_{j}^{2}$. The simulation results based on 500 Monte Carlo tests using 2500 data points each are summarized in Table 5.1.

In agreement with the analysis given in the preceding section, the computational costs with the ILSR algorithm in all the cases considered are reduced quite significantly from those of the ILSNP algorithm and the ILSD algorithm. When $\kappa^{2}$ with a smaller estimation error (e.g., $\hat{\kappa}_{e}^{2}, \hat{\kappa}_{0}^{2}$, or $\hat{\kappa}_{f}^{2}$ ) is utilized, the ILSR algorithm also shows a better accuracy for the parameter estimates than the other ILS-type algorithms in terms of relatively low variance and small RMSE value. It is very interesting to note that the results of ILSR and $\mathrm{ILSR}_{f}$ are almost the same as those of $\mathrm{ILSR}_{0}$. This illustrates that the performance of the ILSR algorithm may not be affected by a slight error in the information about the noise variance ratio $\kappa^{2}$. Moreover, even in the presence of fairly serious error with the noise variance ratio (e.g., $8.8 \%$ in $\hat{\kappa}_{c}^{2}$ and $10.1 \%$ in $\hat{\kappa}_{h}^{2}$ ), the results given by $\operatorname{ILSR}_{c}$ and $\mathrm{ILSR}_{h}$ are still quite acceptable, especially as far as the corresponding RMSE values are concerned. These observations not only have confirmed that the ILSR algorithm can achieve a much improved performance, but also have justified the practical applicability of the ILSR algorithm. 
100 Algorithm for autoregressive signals subject to noise

Table 5.1. Simulation results ( $\mathrm{SNR} \approx 10 \mathrm{~dB}, 2500$ samples, 500 Monte Carlo tests, NFPT $=$ number of flops per test).

\begin{tabular}{|c|c|c|c|c|c|c|c|c|c|}
\hline Method & $a_{1}$ & $a_{2}$ & $a_{3}$ & $a_{4}$ & $\sigma_{v}^{2}$ & $\sigma_{w}^{2}$ & $\mathrm{RE}$ & RMSE & NFPT \\
\hline \multirow[t]{2}{*}{ LS } & 0.8307 & 0.5342 & -0.0358 & 0.0407 & - & - & $60.05 \%$ & $60.09 \%$ & 70301 \\
\hline & \pm 0.0197 & \pm 0.0271 & \pm 0.0258 & \pm 0.0184 & - & - & & & \\
\hline \multirow[t]{2}{*}{ MYW } & 1.6801 & -1.6064 & 0.8232 & -0.2683 & - & - & $22.40 \%$ & $342.92 \%$ & 14033 \\
\hline & \pm 4.5669 & \pm 4.4074 & \pm 2.7033 & \pm 0.7 & - & - & & & \\
\hline \multirow[t]{2}{*}{ ML } & 1.0498 & -0.9681 & 0.4020 & -0.1 & - & - & $27.13 \%$ & $58.07 \%$ & 842472 \\
\hline & \pm 0.5857 & \pm 0.6901 & \pm 0.4804 & \pm 0.1 & - & - & & & \\
\hline \multirow[t]{2}{*}{ ILSNP } & 1.3440 & -1.3280 & 0.6543 & -0.2374 & 1.0127 & 0.3723 & $0.74 \%$ & $10.71 \%$ & 134295 \\
\hline & \pm 0.0897 & \pm 0.1418 & \pm 0.1263 & \pm 0.0 & \pm 0.1505 & \pm 0.0513 & & & \\
\hline \multirow[t]{2}{*}{ ILSD } & 1.3446 & -1.3288 & 0.6550 & -0.2376 & 1.0105 & 0.3732 & $0.68 \%$ & $10.49 \%$ & 103746 \\
\hline & \pm 0.08 & \pm 0.1 & \pm 0.1 & \pm 0.0 & \pm 0.1404 & \pm 0.0472 & & & \\
\hline \multirow[t]{2}{*}{$\operatorname{ILSR}_{a}$} & 1.4354 & -1.4847 & 0.8008 & -0.3048 & 0.8810 & 0.4004 & $11.24 \%$ & $12.64 \%$ & 92852 \\
\hline & \pm 0.0375 & \pm 0.0 & \pm 0.0 & \pm 0.0 & \pm 0.0355 & \pm 0.0161 & & & \\
\hline \multirow[t]{2}{*}{$\mathrm{ILSR}_{b}$} & 1.4138 & -1.4461 & 0.7640 & -0.2872 & 0.9097 & 0.3955 & $8.27 \%$ & $10.11 \%$ & 92843 \\
\hline & \pm 0.0377 & \pm 0.0 & \pm 0.0745 & \pm 0.0 & \pm 0.0 & \pm 0.0 & & & \\
\hline \multirow[t]{2}{*}{$\mathrm{ILSR}_{c}$} & 1.3933 & -1.4096 & 0.7292 & -0.2707 & 0.9374 & 0.3906 & $5.46 \%$ & $7.99 \%$ & 92832 \\
\hline & \pm 0.0379 & \pm 0.0712 & \pm 0.0747 & \pm 0.0437 & \pm 0.0369 & \pm 0.0154 & & & \\
\hline \multirow[t]{2}{*}{$\operatorname{ILSR}_{d}$} & 1.3738 & -1.3750 & 0.6963 & -0.2552 & 0.9641 & 0.3856 & $2.81 \%$ & $6.48 \%$ & 92819 \\
\hline & \pm 0.0380 & \pm 0.0713 & \pm 0.0747 & \pm 0.0436 & \pm 0.0375 & \pm 0 . & & & \\
\hline \multirow[t]{2}{*}{$\mathrm{ILSR}_{e}$} & 1.3546 & -1.3443 & 0.6687 & -0.2436 & 0.9878 & 0.3799 & $0.50 \%$ & $5.79 \%$ & 92827 \\
\hline & \pm 0.0385 & \pm 0.0715 & \pm 0.0733 & \pm 0.0417 & \pm 0.0381 & \pm 0.0146 & & & \\
\hline \multirow[t]{2}{*}{ ILSR $_{0}$} & 1.3490 & -1.3344 & 0.6593 & -0.2392 & 0.9957 & 0.3784 & $0.26 \%$ & $5.77 \%$ & 92823 \\
\hline & \pm 0.0384 & \pm 0.0714 & \pm 0.0732 & \pm 0.0417 & \pm 0.0383 & \pm 0.0145 & & & \\
\hline \multirow[t]{2}{*}{$\mathrm{ILSR}_{f}$} & 1.3371 & -1.3135 & 0.6396 & -0.2299 & 1.0126 & 0.3750 & $1.85 \%$ & $6.04 \%$ & 92814 \\
\hline & \pm 0.0384 & \pm 0.0712 & \pm 0.0729 & \pm 0.0415 & \pm 0.0386 & \pm 0.0143 & & & \\
\hline \multirow[t]{2}{*}{ ILSR $_{g}$} & 1.3205 & -1.2845 & 0.6123 & -0.2172 & 1.0364 & 0.3701 & $4.07 \%$ & $7.02 \%$ & 92799 \\
\hline & \pm 0.0383 & \pm 0.0709 & \pm 0.0725 & \pm 0.0411 & \pm 0.0391 & \pm 0.0139 & & & \\
\hline \multirow[t]{2}{*}{$\mathrm{ILSR}_{h}$} & 1.3047 & -1.2571 & 0.5865 & -0.2052 & 1.0594 & 0.3653 & $6.17 \%$ & $8.39 \%$ & 92783 \\
\hline & \pm 0.0382 & \pm 0.0705 & \pm 0.0720 & \pm 0.0408 & \pm 0.0395 & \pm 0.0136 & & & \\
\hline \multirow[t]{2}{*}{$\mathrm{ILSR}_{i}$} & 1.2897 & -1.2312 & 0.5622 & -0.1940 & 1.0814 & 0.3604 & $8.15 \%$ & $9.91 \%$ & 92773 \\
\hline & \pm 0.0380 & \pm 0.0700 & \pm 0.0714 & \pm 0.0405 & \pm 0.0399 & \pm 0.0133 & & & \\
\hline \multirow[t]{2}{*}{$\mathrm{ILSR}_{j}$} & 1.2755 & -1.2068 & 0.5394 & -0.1835 & 1.1027 & 0.3557 & $10.01 \%$ & $11.47 \%$ & 92767 \\
\hline & \pm 0.0378 & \pm 0.0695 & \pm 0.0707 & \pm 0.0400 & \pm 0.0403 & \pm 0.0130 & & & \\
\hline de & 1.352 & -1.338 & 0.662 & -0.24 & 1.0 & 0.38 & & & \\
\hline
\end{tabular}

\section{Concluding remarks}

In this paper, a simple algorithm has been proposed to make unbiased parameter estimation of noisy AR signals. The sensitivity problem of the ILS-based estimator with respect to the variation of the noise variance ratio has been investigated. The importance of the work presented in this paper is that when a partial information of the driving noise versus the corrupting noise ( situations, the use of the developed ILSR algorithm can be very appealing with regard to estimation accuracy and numerical requirements. 


\section{Acknowledgment}

This work was supported in part by a Research Grant from the Australian Research Council and in part by a Research Grant from the University of Western Sydney, Australia.

\section{References}

[1] S. M. Kay, Modern Spectral Estimation, Prentice-Hall, New Jersey, 1988.

[2] _ Fundamentals of Statistical Signal Processing: Estimation Theory, Prentice-Hall, New Jersey, 1993.

[3] A. Nehorai and P. Stoica, Adaptive algorithms for constrained ARMA signals in the presence of noise, IEEE Trans. Acoust. Speech Signal Process. 36 (1988), no. 8, 1282-1291.

[4] T. F. Quatieri, Discrete-Time Speech Signal Processing: Principles and Practice, Prentice-Hall, New Jersey, 2001.

[5] H. Sakai and M. Arase, Recursive parameter estimation of an autoregressive process disturbed by white noise, Internat. J. Control 30 (1979), no. 6, 949-966.

[6] M. D. Srinath, P. K. Rajasekaran, and R. Viswanathan, Introduction to Statistical Signal Processing with Applications, Prentice-Hall, New Jersey, 1996.

[7] $\mathrm{H}$. Tong, Autoregressive model fitting with noisy data by Akaike's information criterion, IEEE Trans. Inform. Theory 21 (1975), no. 4, 476-480.

[8] W. X. Zheng, An efficient algorithm for parameter estimation of noisy AR processes, Proc. 30th IEEE International Symposium on Circuits and Systems (Hong Kong, 1997), vol. 4, IEEE Press, New Jersey, June 1997, pp. 2509-2512.

[9] On implementation of a least-squares based algorithm for noisy autoregressive signals, Proc. 31st IEEE International Symposium on Circuits and Systems (Calif, 1998), vol. 5, IEEE Press, New Jersey, June 1998, pp. V-21-V-24.

Wei Xing Zheng: School of Quantitative Methods and Mathematical Sciences (QMMS), University of Western Sydney, Penrith South DC, NSW 1797, Australia

E-mail address: w.zheng@uws.edu.au 


\section{Differential Equations \& Nonlinear Mechanics}

\section{An Open Access Journal}

\section{Editor-in-Chief}

K. Vajravelu

USA

Associate Editors

N. Bellomo

Italy

J. L. Bona

USA

J. R. Cannon

USA

S.-N. Chow

USA

B. S. Dandapat

India

E. DiBenedetto

USA

R. Finn

USA

R. L. Fosdick

USA

J. Frehse

Germany

A. Friedman

USA

R. Grimshaw

UK

J. Malek

Czech Republic

J. T. Oden

USA

R. Quintanilla

Spain

K. R. Rajagopal

USA

G. Saccomandi

Italy

Y. Shibata

Japan

Ivar Stakgold

USA

Swaroop Darbha

USA

A. Tani

Japan

S. Turek

Germany

A. Wineman

USA
Website: http://www.hindawi.com/journals/denm/

Aims and Scope

Differential equations play a central role in describing natural phenomena as well as the complex processes that arise from science and technology. Differential Equations \& Nonlinear Mechanics (DENM) will provide a forum for the modeling and analysis of nonlinear phenomena. One of the principal aims of the journal is to promote cross-fertilization between the various subdisciplines of the sciences: physics, chemistry, and biology, as well as various branches of engineering and the medical sciences.

Special efforts will be made to process the papers in a speedy and fair fashion to simultaneously ensure quality and timely publication.

DENM will publish original research papers that are devoted to modeling, analysis, and computational techniques. In addition to original full-length papers, DENM will also publish authoritative and informative review articles devoted to various aspects of ordinary and partial differential equations and their applications to sciences, engineering, and medicine.

\section{Open Access Support}

The Open Access movement is a relatively recent development in academic publishing. It proposes a new business model for academic publishing that enables immediate, worldwide, barrier-free, open access to the full text of research articles for the best interests of the scientific community. All interested readers can read, download, and/or print any Open Access articles without requiring a subscription to the journal in which these articles are published.

In this Open Access model, the publication cost should be covered by the author's institution or research funds. These Open Access charges replace subscription charges and allow the publishers to give the published material away for free to all interested online visitors.

\section{Instructions for Authors}

Original articles are invited and should be submitted through the DENM manuscript tracking system at http://www.mstracking.com/ denm/. Only pdf files are accepted. If, for some reason, submission through the manuscript tracking system is not possible, you can contact denm.support@hindawi.com.

Hindawi Publishing Corporation

410 Park Avenue, 15th Floor, \#287 pmb, New York, NY 10022, USA
HINDAWI 


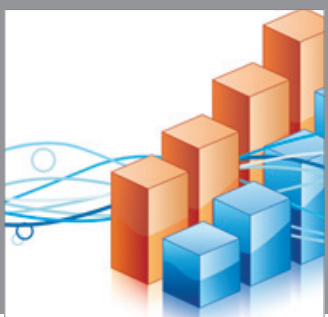

Advances in

Operations Research

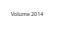

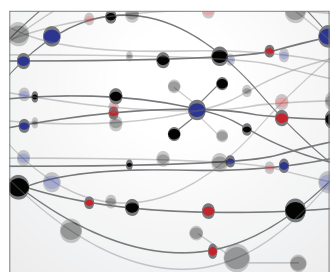

\section{The Scientific} World Journal
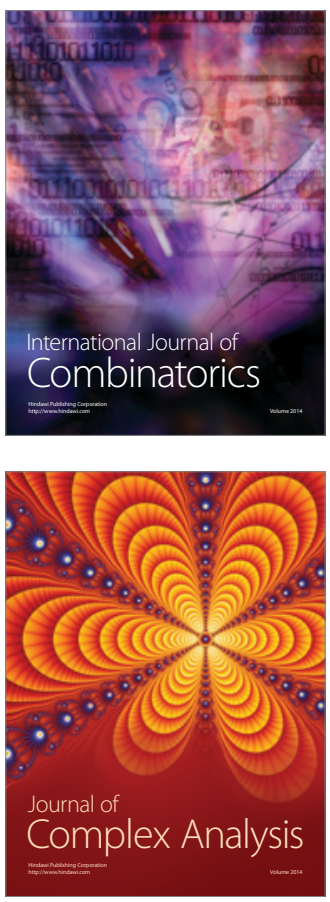

International Journal of

Mathematics and

Mathematical

Sciences
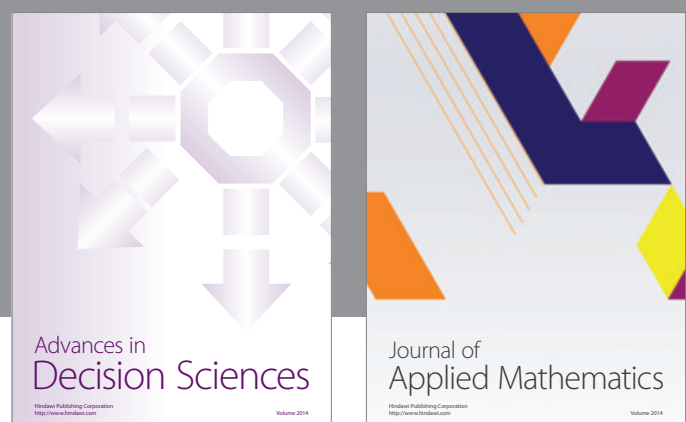

Journal of

Applied Mathematics
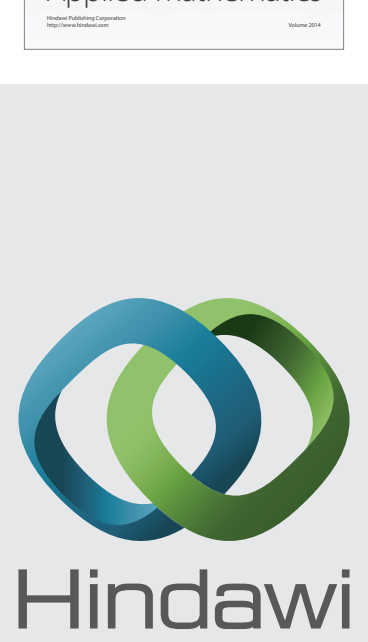

Submit your manuscripts at http://www.hindawi.com
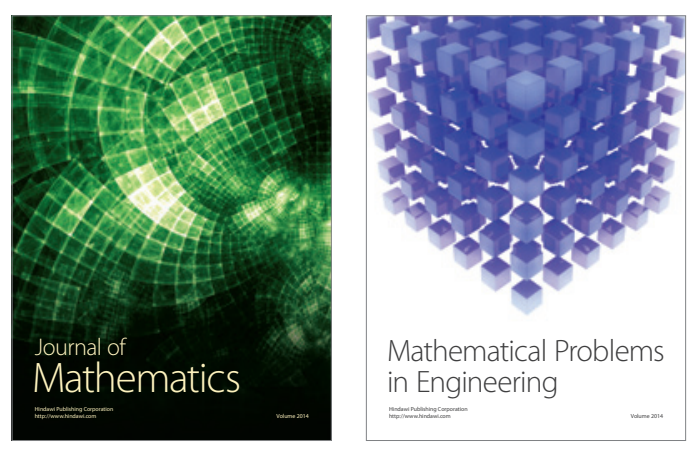

Mathematical Problems in Engineering
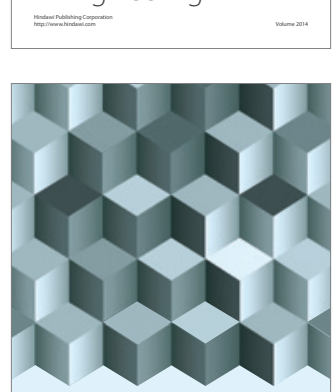

Journal of

Function Spaces
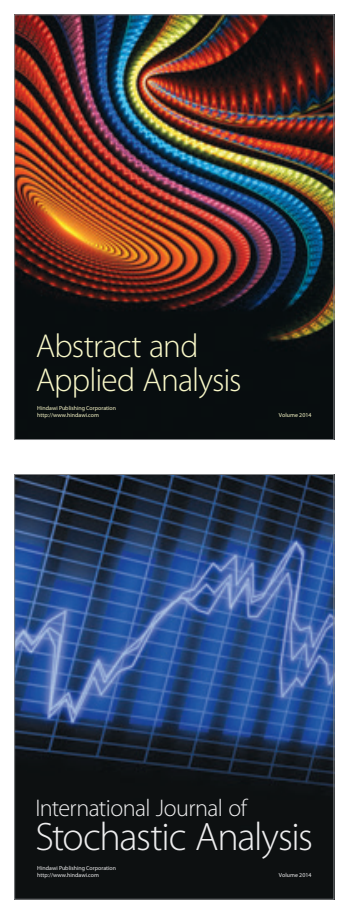

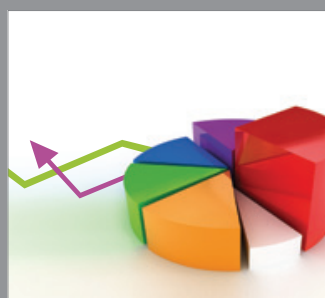

ournal of

Probability and Statistics

Promensencen
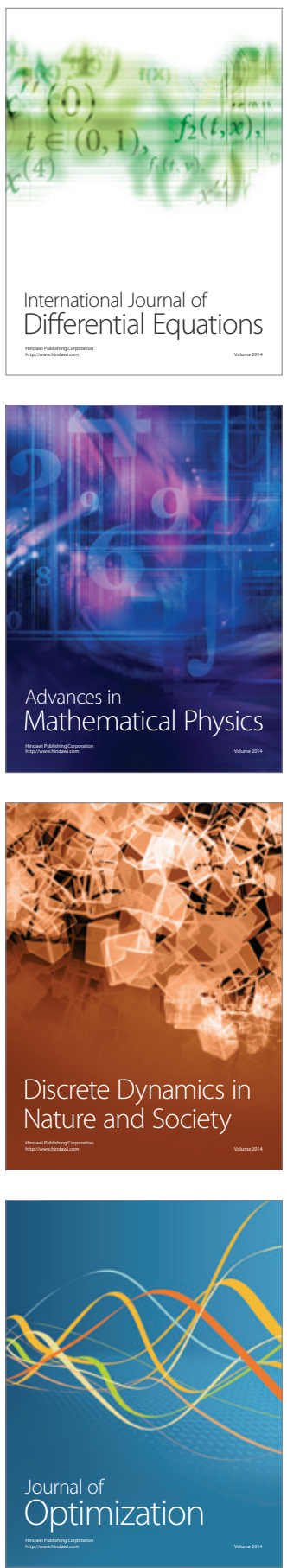